Intervención Polemica

\title{
Cuidar en tiempos de pandemia ${ }^{1}$
}

Karina Inés Ramacciotti

DOI: https://doi.org/10.24215/25457284e126

Universidad Nacional de Quilmes, CONICET, Argentina,

Argentina

karinaramacciotti@gmail.com

(iD http://orcid.org/0000-0002-6724-3926

Recepción: 04 Mayo 2020

Aprobación: 15 Junio 2020

\section{INTRODUCCIÓN}

En un trabajo reciente, Derroteros del Cuidados, sostuvimos que "el cuidado es un vínculo que responde a relaciones de género, familiares, comunitarias, políticas públicas, intervenciones de expertos y profesionales, redes migratorias, y relaciones económicas" (Ramacciotti y Zangaro, 2019). Si hoy tuviéramos que revisar esta conceptualización, sin lugar a duda deberíamos agregar también cómo las situaciones de crisis sanitarias, en este caso la pandemia del coronavirus, han modificado sensiblemente esas relaciones. Esta enfermedad infectocontagiosa, que aún no cuenta ni con tratamiento ni con vacuna, no solo trastocó la ciencia, la investigación, los sistemas de salud y las políticas socioeconómicas, sino que impactó en nuestras vidas cotidianas y relaciones sociales; agudizando aún más la llamada crisis del cuidado que viene denunciado el feminismo.

Los aportes de la Economía Feminista han demostrado cómo aquellas tareas que permiten la reproducción cotidiana y son presentadas de manera "natural", como propias de la naturaleza de las mujeres y que sostienen la dinámica familiar, resultan de un trabajo no pago y además permiten sostener la dinámica del capitalismo. Es decir, tal como lo sostiene Silvia Federici (1971), preparar alimentos, lavar, dar sustento afectivo, organizar la economía familiar, entre otras tareas, además de no ser labores naturales de las mujeres resultan un trabajo no remunerado, en tanto garantizan la reproducción de la fuerza de trabajo y, mediante ellas, se produce valor. La visibilidad de las tareas solo se percibe cuando no se realizan, de lo contrario se normalizan, pierden valor y por lo tanto son pasibles de no ser remuneradas.

Esta renovación en la agenda de las ciencias sociales tuvo impacto en el área político institucional en la Argentina cuando el presidente Alberto Fernández instituyó a fines del 2019 el Ministerio de las Mujeres, Géneros y Diversidad con la dirección de la ministra Elizabeth Gómez Alcorta. Este nuevo ministerio tiene como una de sus prioridades trabajar por una "redistribución más justa de las tareas de cuidado ya que se reconoce que es un aspecto históricamente invisibilizado, social y económicamente, y librado a decisiones en el marco de la esfera privada de las familias" (Ministerio de las Mujeres, Géneros y Diversidad, 2020).

A partir de los primeros casos de coronavirus, en marzo del 2020, el término "cuidar" tomó un protagonismo inusitado. Varios enunciadores lo tomaron como propio: el slogan "Quedate en casa, nosotros te cuidamos", utilizado como una forma de transmitir que el cuidado individual colabora en la reducción de contagios; el dispositivo "CuidAR", nueva aplicación del gobierno nacional para autodiagnóstico y rastreo del Covid-19; las empresas de medicina privada también han manejado el concepto para posicionar sus marcas en el mercado. Entonces, frente a una mayor presencia de esta narrativa nos preguntamos si esta abundancia del término, centrado en la responsabilidad individual, no está vaciando el contenido social y colectivo que el mismo porta. Sospechamos que se ha caído en una romantización y banalización del término y se están invisibilizado, por lo menos, dos cuestiones que quisiéramos desarrollar en esta oportunidad. Primero, cómo 
afectó a las mujeres esta pandemia sobrecargando sobre ellas las tareas domésticas y de cuidado; y cómo se conmovieron las condiciones laborales de quienes cuidan de manera profesional, por ejemplo en el sector de enfermería. Para desarrollar estas ideas vamos a remitirnos a la historia y revisar algunos hitos sobre el tema en cuestión que nos permitan analizar la trama social e histórica del cuidado.

\section{Madres que Cuidan como Destino esencializado}

Desde el siglo XX, en la Argentina los discursos médicos y las políticas públicas estimularon el rol de las mujeres en cuanto su capacidad de engendrar hijos "fuertes y sanos para el futuro" y transformarse en "Reinas del hogar" (Nari, 2005; Lobato, 2007). Dentro de este contexto las prácticas de higiene, que ellas podrían estimular desde el hogar, fueron entendidas como cuasi obligaciones. Incluían prácticas de limpieza, mantenimiento del orden, preparación meticulosa de la comida y cuidados a hijes y personas que requiriesen cuidados. Estas actividades, diarias y rutinarias, las ponían en un lugar central para evitar focos infecciosos y mantener la salud de la infancia. En aquellos casos que la "falta de moralidad" y las costumbres "inadecuadas" atentaban contra dicho rol esencializado de cuidado, la escuela era considerada como el lugar indicado para instruir sobre pautas modernas de higiene y alimentación y, en un escenario idealizado, serían los infantes quienes llevarían esas conductas a los hogares (Ramacciotti, 2020). Tal como señalan Carolina Biernat y Graciela Queirolo (2018), a las mujeres en cuanto madres se consideraba que poseían naturalmente ciertos atributos emocionales y físicos: la paciencia, la ternura o el amor, la predisposición para realizar tareas rutinarias, la abnegación o el sacrificio. Era esta naturaleza femenina la que se dispensaba dentro del mundo doméstico y les permitía a las mujeres gestionar y ejecutar los múltiples y permanentes quehaceres de la casaasear, ordenar, abastecer, zurcir, cocinar-, que implicaban asistir y cuidar a otras y otros integrantes del grupo familiar. Así, las mujeres ocuparon empleos en los que desplegaron esa supuesta naturaleza femenina que las hacía eficientes en dichas labores.

Para las mujeres que debían trabajar el Estado, por medio del diseño y la implementación de políticas públicas, trazó un conjunto de políticas sociales que, desde 1907, intentaron resguardar la capacidad reproductiva de las mujeres. La premura estuvo dada por velar la salud del útero de aquellas trabajadoras que tuvieran capacidad reproductiva activa y por intentar que su actividad laboral no lesionara la salud de la mujer solo en cuanto a su capacidad de reproducir. La reducción de las horas que ellas deberían trabajar, el estímulo para que los patrones colocaran sillas en las fábricas para que se pudieran sentar y así no lesionar sus órganos reproductivos, las licencias por maternidad, el seguro de maternidad, la instalación de guarderías en los lugares de trabajo para estimular el amamantamiento y el cuidado materno fueron medidas dirigidas a las mujeres jóvenes en tanto se preservaba sus capacidades reproductivas y una crianza "responsable". Sabido es que los empleadores supieron cómo evitar su cumplimiento y el Estado no se esforzó políticamente en la supervisión de su ejecución; pero las demandas de su cumplimiento continuaron vigentes dentro de los movimientos de mujeres y las licencias por paternidad aún siguen estando en el terreno de la retórica política y hasta el momento no ha habido modificaciones sustantivas al respecto (Lobato, 2007; Biernat y Ramacciotti, 2013; Queirolo, 2019).

¿Qué nos trajo el COVID 19? Las encuestas sobre el uso del tiempo y trabajo no remunerado han demostrado de manera cuantitativa que ellas dedican mucho más tiempo que los varones a las tareas domésticas y al cuidado, independientemente del hecho de trabajar además de forma remunerada. En línea con lo que postula Karina Brovelli (2019, p.31) "si nos propusieran representar el cuidado, posiblemente elegiríamos imágenes de tareas tales como la preparación de comidas, el control de la fiebre y la administración de medicación a algún familiar, la ayuda a les niñes en las tareas escolares, entre muchas otras actividades similares. Ahora bien, si profundizamos la mirada, probablemente encontraremos que, en todas esas imágenes, las personas encargadas del cuidado son mujeres". 
La decisión de cerrar las escuelas y los centros educativos terapéuticos como medida de prevención del contagio, sin una política que contemplara qué personas iban a enseñar o cuidar de manera profesional a les niñes o personas con discapacidad durante este período, demuestra la ausencia de una mirada atenta a la perspectiva de género y una tendencia a la familiarización del cuidado. También, se invisibiliza el reconocimiento de que para enseñar y cuidar de forma profesional se necesitan técnicas y saberes particulares y específicos. Por más que parezca obvio es importante señalar que no cualquier persona puede enseñar ni dar los apoyos necesarios para que se pueda mantener una vida autónoma. Que ambas tareas demandan conocimientos, técnicas y una reflexión sobre la práctica que en estas situaciones se diluye. Entonces, esta crisis sanitaria visibiliza la ausencia de programas de cuidados y de medidas que coordinan de manera equitativa la vida laboral y personal; regresa, aun con más fuerza, una mirada moralizadora de quienes portan condiciones "naturales" para cuidar.

Desde el Observatorio de Género y Políticas Públicas de la Universidad Nacional José Clemente Paz, se llevó adelante durante la tercera semana de cuarentena en el Gran Buenos Aires, un relevamiento sobre las relaciones de género en cuestiones de cuidado. En las respuestas relevadas, se encontraron que entre las tareas que se les incrementaron a las mujeres están las de limpieza, paralelamente al acompañamiento de las tareas escolares. La mayoría de las personas encuestadas respondieron que una de las "nuevas" actividades que comenzaron a hacer en el hogar durante el periodo de pandemia fue el armado y/o seguimiento de clases virtuales. Asimismo, se observó que los varones, durante la cuarentena, además de estas tareas, dedican tiempo a realizar deportes en el hogar, a entretenimientos como videojuegos y programas de televisión, o incluso a la lectura. Mientras que las mujeres plantearon que tuvieron un aumento de dedicación considerable en las tareas básicas de reproducción y un mínimo incremento en las actividades de recreación antes mencionadas. Según este informe, la educación se instaló y recargó al ámbito de lo "privado", "espacio al que las mujeres han sido asignadas, material y simbólicamente, a lo largo de la historia; espacio ampliamente romantizado" (Goren, Jerez y Figueroa, 2020, pp. 8-9).

Tomando como ejemplo el ámbito académico y los datos que han surgido en una nota periodística titulada "Women academics seem to be submitting fewer papers during coronavirus. 'Never seen anything like it,' says one editor", ya hay indicios en la academia norteamericana que demuestran que, desde los inicios de la pandemia y con las medidas de aislamiento implementadas, las revistas científicas han recibido un 50 por ciento más de artículos científicos de varones que de mujeres. Mientras que ellas han tenido que repartir su tiempo entre las tareas de cuidado y laborales, ellos pareciera que cuentan con mayor tiempo para la investigación dado que sus mujeres cuidan a sus hijes. Entonces, si bien estamos en el terreno de las conjeturas, existen algunos indicios para sostener que el confinamiento y las exigencias de cuidado que tienen las mujeres, profundizan las brechas en la producción académica entre hombres y mujeres, y todo pareciera revelar que el techo de cristal seguirá estando bastante firme en los próximos años. ${ }^{2}$

Claro está que ambas referencias se vinculan con mujeres de clase media, con trabajo y ciertos derechos, que en este contexto parecen privilegios, como el de tener un salario mensual, una vivienda, electricidad y conectividad tecnológica. Nada dice de cómo los efectos de esta pandemia afectan a mujeres de sectores populares y de las múltiples dificultades que impuso el aislamiento para quienes están imposibilitadas de movilizarse para conseguir algún ingreso diario, quienes sufren la violencia de género potenciada por el encierro o quienes deben salir a trabajar porque sus tareas están exceptuadas y no cuentan con una red de cuidados para sus hijes. Entonces, los estudios sociales del cuidado nos permiten ver otras formas de la desigualdad, que se basan en el género, pero que también se vinculan con variables de clase, étnicas y generacionales, entre otras. 


\section{Cuidado Profesional}

Cuidar denota un fenómeno multidimensional, dinámico y complejo, con componentes científicotecnológicos orientados hacia la dimensión biológica de la persona cuidada y componentes humanísticos orientados hacia la dimensión emocional, intelectual, social, cultural y espiritual. Asimismo, y retomando la idea de Francisca Pereyra y Ariela Micha (2016), las y los trabajadores del cuidado desempeñan actividades que contribuyen a la salud, la seguridad física, y el desarrollo de habilidades cognitivas, físicas o emocionales de las personas, y que interactúan directamente con los receptores del servicio en cuestión. Típicamente, estas ocupaciones incluyen docentes de todos los niveles, terapeutas, médicas/os y enfermeras/os, y se concentran en el sector salud y en el de educación.

Entonces, es muy variado el sector laboral vinculado a quienes cuidan de manera profesional. En esta intervención nos vamos a concentrar en el trabajo del sector de enfermería. Sabido es que en las narrativas las enfermeras no forman parte del "Panteón" de la medicina. La historia de la medicina suele estar cargada de relatos de trayectorias de galenos en los que se destacan sus virtudes y logros, tanto en el terreno de la ciencia como en el de la política. Estos relatos laudatorios han contribuido a la difusión de información básica sobre ciertas figuras "relevantes", pero suelen obviar a otros personajes que tuvieron un rol destacado en la especialización de funciones de las agencias sanitarias y quedaron al margen tanto de las historias oficiales como de aquellas que se proponen diferenciarse con una mirada más crítica y renovada. Me refiero tanto las médicas como el amplio abanico de tareas sociosanitarias que estuvieron en manos de las mujeres y están invisibilizadas. Es una historia que implica la construcción de un proceso fascinante, que incluye a los que están en los hospitales, atrás o al costado de los facultativos más descollantes; a sus "auxiliares" en los laboratorios, a funcionarias que completan rutinariamente formularios, informes, historias clínicas para la administración de la gestión de la investigación, o para la salud. Muchas son mujeres, como las visitadoras de higiene y su papel se refleja a veces en textos burocráticos, o debe ser imaginado a través de otras escrituras, justamente, las de quienes planifican las políticas y dependen de aquellas para su ejecución. El Estado requiere de estas personas para proveer vacunas, brindar cuidados, asistencia y medicinas, mientras que de su esfuerzo, comprensión y afecto se pueden detectar dolencias invisibles ante los ojos de la mirada de la clínica médica. Pero, que sean labores que han tendido a la feminización no implica que dichas tareas sean un patrimonio natural de ellas, sino que responden a una construcción histórica y socialmente impuesta, que determinó entre otras cosas, las diferencias y las desigualdades que la caracterizan respecto de otras profesiones no feminizadas.

En los grandes centros urbanos de la Argentina, las tareas de cuidado y asistencia hacia las personas que demandaban atención en el sistema sanitario estuvieron ligadas a las labores que las mujeres brindaron, dadas sus supuestas virtudes vinculadas a la caridad y al cuidado, en las cuales podían desplegar sus dotes innatas. Las urgencias que motivaban los brotes epidémicos, los accidentes laborales y las enfermedades infantiles, entre otras cuestiones, solían tenerlas como principales dadoras de atención y cuidado. En las salas de los hospitales fue habitual la presencia de las Hermanas de la Caridad, religiosas de otras órdenes y mujeres de sectores populares que se acercaban a los hospitales y ejercían como cuidadoras, enfermeras, mucamas, voluntarias o secretarias. El atractivo, para las segundas, podía ser tanto un exiguo salario, la esperanza de obtener, en algún momento, un cargo efectivo, la entrega de alguna propina, o tener un techo y comida. Las religiosas tenían una mayor jerarquía, ya que organizaban las salas y podían dar órdenes directas al personal que allí trabajaba. Con excepción de algunos de los hospitales administrados por comunidades inmigrantes, que contaban con cursos de enfermería o pequeñas escuelas como, por ejemplo, el Hospital Británico, no existían ámbitos, ni bajo la tutela estatal ni universitaria, para la formación profesional (Ramacciotti, 2020).

Estas actividades, al estar guiadas por la lógica del sacrificio y la caridad, fueron argumentos funcionales para mantener sueldos bajos o ad honorem. En efecto, al considerar esas intervenciones como propias de sus condiciones naturales, se produjo una desvalorización de sus tareas y, como consecuencia, un menosprecio 
a su capacitación y a sus derechos laborales. La concepción del trabajo femenino como complementario al presupuesto familiar sostenido por los varones también reforzó el mantenimiento de salarios inferiores.

En este primer momento los espacios formativos tendieron a orientarse exclusivamente a una formación práctica, focalizada en las tareas de limpieza, cuidado, atención de los primeros auxilios y el estricto acatamiento de las prescripciones médicas. Un mojón importante de la modernización del cuidado en la ciudad de Buenos Aires estuvo ligado al impulso que produjo Cecilia Grierson, primera graduada en medicina de la Universidad de Buenos Aires (1889). Esas clases estuvieron destinadas al personal que ejercía en los hospitales porteños y algunas de ellas tuvieron lugar en los consultorios de los colegas de Grierson que apoyaron su iniciativa pues consideraban necesario formar profesionales en enfermería o, al menos, instruir al personal que se ocupaba de esas tareas. Estas clases fueron el inicio de la Escuela de Enfermeras, Enfermeros y Masajistas de la Ciudad de Buenos Aires (Martin, 2015).

A este conjunto de espacios formativos debe sumarse el creado en la Universidad de Buenos Aires en 1924. La Escuela de Nurses, impulsada al interior del Instituto de Medicina Experimental, fue un espacio de capacitación feminizado para las dolencias crónicas. El personal a cargo del cuidado debería contar con una formación específica. No obstante, el salario de estas mujeres, quienes debían cursar materias por dos años en un sistema de internado, oscilaba entre 80 a 120 pesos. Este sueldo se asemejaba al que cobraban otros trabajadores tales como, por ejemplo, los peones, el portero y el sereno. Los médicos podían cobrar entre 230 a 1500 pesos según su jerarquía. Este hallazgo da cuenta de uno de los dilemas más importantes dentro de la profesión: cómo estimular espacios de capacitación y perfeccionamiento ante patologías cada vez más complejas cuando las brechas salariales son tan amplias (Buschini, 2020).

En las décadas de 1940 y 1950 la expansión de puestos laborales vinculados a las tareas de cuidado y de enfermería se motivaron por la expansión hospitalaria, el incremento de las campañas sanitarias y la modernización en las técnicas médicas que demandaban mayores tareas en su implementación. Si en la etapa anterior la prioridad en la formación fue la emergencia sanitaria, los primeros auxilios y la atención hospitalaria, en esta segunda etapa la asistencia se articuló con la faz preventiva y la preparación profesional para salir de las unidades hospitalarias y ocuparse tanto de la prevención como de la atención y derivación a los centros hospitalarios. El eje de atención fue tanto los espacios urbanos como los rurales y, por lo tanto, a las preocupaciones de las enfermedades epidémicas e infectocontagiosas se sumaron las inquietudes por las enfermedades endémicas y crónicas. Durante estos años las políticas sanitarias estimularon espacios formativos para incrementar dichas pericias. A los espacios formativos ya existentes se sumaron La escuela de Enfermería de la Secretaría de Salud Pública (1947) y la Escuela de Enfermeras de la Fundación Eva Perón (1948), que colaboraron en capacitar recursos humanos para satisfacer las demandas de un sistema de salud pública más complejo (Ramacciotti y Valobra, 2015).

A partir de los años cincuenta y, a tono con lo que sucedía en otras latitudes, la enfermería pasó a tener un lugar en la vida universitaria. Se mejoraron las técnicas a la luz de los cambios que se producían en la ciencia, la tecnología y en la industria farmacéutica. Se modificaron los programas de estudio, se incrementaron las horas de cursadas, se mantuvieron estrictas normas morales ya que, según lo que se creía, el profesionalismo no solo pasaba por manejar prácticas modernas de asistencia y cuidado, sino por contar con pautas de obediencia y de disciplina que iban desde el uso del uniforme, hasta el mantenimiento de conductas consideradas ideales en la vida privada.

Entonces el tránsito por la vida universitaria trajo como consecuencia para muchas de ellas un salto cualitativo, ya que implicó una forma de movilidad, de ascenso social, jerarquizó a la profesión e inspiró a que muchas de las universitarias tuvieran un papel importante, tanto en la representación de la enfermería nacional como internacional; empezaran a ocupar cargos docentes y de supervisión en los espacios formativos $\mathrm{y}$ en las agencias estatales, tanto provinciales como nacionales, y tareas de edición en revistas profesionales. Como contrapartida, la existencia de enfermeras universitarias en los ambientes laborales amplió la brecha 
entre las graduadas en enfermería y potenció, aún más, diferencias regionales, intergeneracionales y de clase (Ramacciotti y Valobra, 2017).

A partir de los años noventa se reglamentó el ejercicio profesional y se delimitaron injerencias e incumbencias de la práctica profesional y laboral, tal como como la extensión de la jornada a seis horas por ser considerada tarea insalubre (Faccia, 2015). Pero, como suele suceder en los procesos históricos, muchos de estos avances suelen tener ciertos retrocesos como el sucedido a fines del 2018. Entonces, el gobierno de Cambiemos aprobó en la Legislatura de Ciudad de Buenos Aires una norma que pretendía excluir a los enfermeros del equipo de salud, considerándolos "personal administrativo". Luego de una activa movilización esta normativa quedó sin efecto.

En la actualidad la profesión, según un estudio reciente, está marcada por salarios bajos, pluriempleo, estrés laboral y falta de equipamiento e infraestructura adecuados, exposición a riesgos biológicos, químicos y físicos, con patologías osteomusculares y articulares producidas por esfuerzos físicos que demandan las tareas, así como también alteraciones del patrón de sueño y fatiga, sobre todo entre las mujeres que trabajan en horarios nocturnos y deben compatibilizar el trabajo con las responsabilidades domésticas. Asimismo, la falta de recursos humanos conforma uno de los problemas fundamentales que afectan a la ocupación, y este déficit de trabajadores se manifiesta no solo por la escasez en el número de enfermeras/os en ejercicio y la consecuente sobrecarga laboral que esto implica, sino también por su calificación, conformando un déficit cuali-cuantitativo de recursos humanos (Pereyra y Micha, 2016).

Estas situaciones estructurales se ven avivadas en el contexto pandémico. Si bien se reconoce desde el titular de un periódico que "Los enfermeros, el recurso humano imprescindible para la lucha contra el coronavirus" y que la Organización Mundial de la Salud ha declarado el 2020 Año de la Enfermería por ser el aniversario de Florence Nightingale, ${ }^{3}$ dichas afirmaciones y celebraciones no aminoran las situaciones de discriminación que ha sufrido el personal de salud, como el caso varias veces repetido de enfermeras que han recibido amenazas de sus vecinos y fueron acusadas de ser agentes transmisores del virus. Tampoco es un hecho pasajero que la primera víctima de coronavirus dentro de los profesionales de la salud haya sido un enfermero de 47 años del Instituto Médico Brandsen. Ese "primer lugar de las trincheras", como se suele decir, homologando al ámbito sanitario como a un campo de batalla, los expone a un lugar de mayor contagio. Se suele destacar la "vocación”, el "amor hacia el otro", "su actitud de entrega” por sobre las concretas condiciones de trabajo que interpelan a la profesión. La falta de insumos, de capacitación, de materiales de protección adecuadas, son situaciones que, si bien no son nuevas, ante una epidemia toman un mayor protagonismo en la vida hospitalaria, que no siempre es visible.

Entonces, si retomamos las palabras iniciales nos preguntamos si habría que tener cierta cautela ante las narrativas que utilizan el "cuidado" y lo asocian al terreno de la responsabilidad individual, perdiendo la connotación colectiva y política que debería portar. Pensar el cuidado en tiempos de pandemia no debería perder de vista la intersección entre las relaciones sociales y de género, y las políticas públicas deberían reforzar responsabilidades de quién y cómo lo provee para reducir las brechas de desigualdad existente.

A partir de estas ideas, nos hacemos algunas preguntas que aún están en un panorama incierto ¿̇las licencias por maternidad (y en algunos casos por enfermedad de familiares) se ven modificadas por el trabajo virtual o por trabajos en situaciones de excepción? O, dicho de otra forma, ¿los derechos laborales adquiridos se ven modificados por el trabajo virtual y la emergencia sanitaria?, ¿las políticas de investigación y ciencia tendrán alguna respuesta institucional para que, en este contexto de recarga de trabajo, las formas de "medir" la productividad tengan una mirada de género que contemple las desigualdades provocadas en este contexto de trabajo?, ¿habrá un debate en torno al mejoramiento de las condiciones laborales para los trabajadores de la salud que los corra del lugar de "héroes silenciosos" o del "primer lugar en la trinchera" y se mejore las condiciones salariales y laborales? El panorama es incierto, pero las preguntas quedan abiertas para proseguir el debate. 
Entonces estemos alerta ante las situaciones que en nombre del cuidado erosionan derechos laborales, justifican acciones de discriminación, promueven el abandono de tratamientos terapéuticos y biologizan roles que son sociales y culturales y no determinados por el sexo. Dicho de forma sintética, cuidar no debe habilitar situaciones de abusos de poder ni de violación de derechos adquiridos, sino que debe potenciar formas solidarias, afectivas y profesionales que evidencien la autonomía y las relaciones políticas y sociales que atraviesan el concepto.

\section{REFERENCIAS}

Biernat, C. y Queirolo, G. (2018). Mujeres, profesiones y procesos de profesionalización en la Argentina y Brasil. Anuario del Instituto de Historia Argentina, 18(1), e060. https://doi.org/10.24215/2314-257Xe060

Biernat, C. y Ramacciotti, K. (2013). Crecer y Multiplicarse. La politica sanitaria materno infantil. Argentina, 1900-1960. Buenos Aires: Biblos.

Brovelli, K. (2019). El cuidado: una actividad indispensable pero invisible. En G. Guerrero; K. Ramacciotti y M. Zangaro (Comps.), Los derroteros del cuidado. Bernal: Universidad Nacional de Quilmes (pp. 31-45). Recuperado de: https://deya.unq.edu.ar/publicaciones/cuidado/

Buschini, J. (2020). La "Escuela de Nurses" del Instituto de Medicina Experimental. En K. Ramacciotti (Dir.), Historias de la enfermería en Argentina. Pasado y Presente de una profesión (pp. 99-128). José C. Paz: Editorial de la Universidad Nacional de José C. Paz.

Faccia, K. (2015). Continuidades y rupturas del proceso de profesionalización de la enfermería (1955-2011). En C. Biernat, J. Cerdá y K. Ramacciotti (Dir.), La salud pública y la enfermeria en la Argentina (pp. 315-331).Bernal: Universidad Nacional de Quilmes.

Federici, S. [1971] (2018). Contraatacando desde la cocina. En El patriarcado del salario (pp. 21-42). Buenos Aires: Tinta Limón.

Goren, N., Jerez, C. y Figueroa, Y. (2020). ¿Los cuidados en agenda? Reflexiones y proyecciones feministas en época de COVID-19. Informe Desigualdades en el marco de la Pandemia. Recuperado de https://www.clacso.org/wpcontent/uploads/2020/04/IESCODE-Desigualdades-en-el-marco-de-la-pandemia.pdf

Lobato, M. (2007). Historia de las trabajadoras en la Argentina (1869-1960). Buenos Aires: Edhasa.

Martin, A. (2015). Mujeres y enfermería: una asociación temprana y estable (1886-1940). En C. Biernat, J. Cerdá y K. Ramacciotti (Dirs.). La salud pública y la enfermería en la Argentina (pp. 257-286). Bernal: Editorial de la Universidad Nacional de Quilmes.

Ministerio de las Mujeres, Géneros y Diversidad (2020). Cuidados. Recuperado de https://www.argentina.gob.ar/ge neros/cuidados

Nari, M. (2005). Politicas de maternidad y maternalismo politico. Buenos Aires (1890-1940). Buenos Aires: Biblos.

Pereyra, F. y Micha, A. (2016). La configuración de las condiciones laborales de la enfermería en el Área Metropolitana de Buenos Aires: un análisis en el cruce del orden de género y la organización del sistema de salud. Salud Colectiva, 12(2), https://dx.doi.org/10.18294/sc.2016.730

Ramacciotti, K. (2020). El cuidado sanitario. Hacia una historia de la enfermería en Argentina. En K. Ramacciotti (Dir.), Historias de la enfermería en Argentina. Pasado y Presente de una profesión (pp. 31-65). José C. Paz: Editorial de la Universidad Nacional de José C Paz.

Ramacciotti, K y Valobra, A. (2015). Feminización y profesionalización de la enfermería. En C. Biernat, J. M. Cerdá y K. Ramacciotti (Dirs.), La salud pública y la enfermería en la Argentina (pp. 287-313). Bernal: Universidad Nacional de Quilmes.

Ramacciotti, K. y Valobra, A. (2017). El dilema Nightingale: controversias sobre la profesionalización de la enfermería en Argentina 1949-1967. Dynamis, 37(2), http://dx.doi.org/10.4321/S0211-95362017000200006 
Ramacciotti, K. y Zangaro, M. (2019). Presentación. En G. Guerrero, K. Ramacciotti y M. Zangaro (Comps.), Los derroteros del cuidado (pp. 7-17). Bernal: Universidad Nacional de Quilmes. Recuperado https://deya.unq.edu .ar/publicaciones/cuidado/.

Queirolo, G. (2019). El cuidado en la Historia: nuevos análisis para un antiguo problema. En G. Guerrero; K. Ramacciotti y M. Zangaro (Comps.). Los derroteros del cuidado (pp. 45-58). Bernal: Universidad Nacional de Quilmes. Recuperado de https://deya.unq.edu.ar/publicaciones/cuidado/

\section{Notas}

1 Esta intervención se vincula con las discusiones teóricas y debates propiciadas en el proyecto NA00317 aprobado por Resolución $N^{\circ} 200$ del 31 de mayo de 2017 de la Universidad Nacional de José C. Paz; en el proyecto de investigación "El proceso de profesionalización del cuidado sanitario. La enfermería universitaria en Argentina (1940-1970)", financiado por la Universidad Nacional de Quilmes ( $\left.\mathrm{N}^{\circ} 1290 / 19\right)$, en el proyecto "Modernización en clave de género (H846)" y en el proyecto "Género y modernización política (Argentina, 1955-1970)", Proyecto Plurianual, CONICET. Asimismo, agradezco la invitación a participar en "Diálogos sobre el cuidado; experiencias, reflexiones e investigaciones" organizado por María Pía Venturiello y Karina Guerscberg el 29 de abril de 2020 bajo la modalidad teleconferencia. Disponible en https://www.facebook.com/SenderosDelSemb/videos/883330045428140/UzpfSTEwOTU2ODc5MTA6MTA yMjA5MDQ1MTExNzc5NjM/?fref=search\&_tn_=\%2Cd\%2CP-R\&eid=ARBDtC5ZBEhpziO4niJGMsz-TluE utMd-DHOSE-v1DSgujzZx4FfmqBcxF9kDoCcpU7-u2hz5T8hWY0g

2 https://www.thelily.com/women-academics-seem-to-be-submitting-fewer-papers-during-coronavirus-never-seen-any thing-like-it-says-one-editor/?fbclid=IwAR31eOBTFpVTY8LTWwlEl3sxqw63PmKkGQnOQZySeY1HiZv1Mob UI4HofpQ

3 Enfermera que en la Guerra de Crimea (1853) modificó las técnicas de cuidados hacia las personas heridas y permitió reducir los índices de mortalidad por infecciones. Su método se basó en la ventilación y en una estricta desinfección ambos procesos rigurosamente controlados y cuantificados para poder medir su impacto. Es considerada una precursora de la estadística sanitaria en la enfermería moderna y en el feminismo inglés.

\section{BY-NC-SA}

